\title{
Cosmological Analysis of Dynamical Chern-Simons Modified Gravity via Dark Energy Scenario
}

\author{
Abdul Jawad and Shamaila Rani \\ Department of Mathematics, COMSATS Institute of Information Technology, Lahore 54000, Pakistan \\ Correspondence should be addressed to Abdul Jawad; jawadab181@yahoo.com
}

Received 19 June 2015; Revised 19 August 2015; Accepted 20 August 2015

Academic Editor: George Siopsis

Copyright ( 2015 A. Jawad and S. Rani. This is an open access article distributed under the Creative Commons Attribution License, which permits unrestricted use, distribution, and reproduction in any medium, provided the original work is properly cited. The publication of this article was funded by SCOAP S $^{3}$

\begin{abstract}
The purpose of this paper is to study the cosmological evolution of the universe in the framework of dynamical Chern-Simons modified gravity. We take pilgrim dark energy model with Hubble and event horizons in interacting scenario with cold dark matter. For this scenario, we discuss cosmological parameters such as Hubble and equation of state and cosmological plane like $\omega_{9}-\omega_{9}^{\prime}$ and squared speed of sound. It is found that Hubble parameter approaches the ranges $75_{-0.5}^{+0.5}$ (for $\left.u=2\right)$ and $(74,74.30)($ for $u=1,-1,-2)$ for Hubble horizon pilgrim dark energy. It implies the ranges $74.80_{-0.005}^{+0.005}($ for $u=2)$ and $(73.4,74)$ (for $\left.u=-2\right)$ for event horizon pilgrim dark energy. The equation of state parameter provides consistent ranges with different observational schemes. Also, $\omega_{9}-\omega_{9}^{\prime}$ planes lie in the range $\left(\omega_{\vartheta}=-1.13_{-0.25}^{+0.24}, \omega_{\vartheta}^{\prime}<1.32\right)$. The squared speed of sound shows stability for all present models in the present scenario. We would like to mention here that our results of various cosmological parameters show consistency with different observational data like Planck, WP, BAO, $H_{0}$, SNLS, and WMAP.
\end{abstract}

\section{Introduction}

The astronomers have put up a struggle in the subject of cosmology and pointed out that the rapid expansion of the universe is because of an unknown force dubbed as dark energy (DE) with the help of different cosmological and astronomical data (arising from well-known observational schemes) [1-7]. This DE possesses repulsive force but its nature is still unknown. Unfortunately, any solid argument in favor of DE candidate has not been given till today. The pioneer candidate of $\mathrm{DE}$ is the cosmological constant but it is plagued by two serious problems such as fine-tuning and cosmic coincidence. Different setups have been adopted, dynamical DE models, modified and higher dimensional gravity theories, and effective description of the models and gravity theories, in order to illustrate its unknown nature and avoid the cosmological constant problems. The family of Chaplygin gas [8-10], holographic [11, 12] and new agegraphic $\mathrm{DE}$ [13], pilgrim [14-21], and so forth lie in the category of the dynamical DE models which are being used most commonly in explaining the cosmological scenario.
Among all dynamical models, holographic DE (HDE) model has been constructed in the framework of quantum gravity on the basis of holographic principle [22]. This model has a remarkable feature because it links the DE density to the cosmic horizon $[23,24]$ and has been tested through various astronomical observations [25]. It is suggested that this model may play a crucial role in solving $\mathrm{DE}$ issues up to some extent. The black hole (BH) entropy plays an essential role in the derivation of this $\mathrm{DE}$ model. On the basis of $\mathrm{BH}$ entropy relation, Cohen et al. [26] set a relation between the ultraviolet cutoff (short distance) and infrared (IR) cutoff (long distances) by approximating the limit on the formation of black hole in the quantum gravity. It means that total energy of the system with size $L$ should not exceed the mass of a $\mathrm{BH}$ with the same radius. For the largest value of $L$ to saturate this process, the energy density of HDE is given by

$$
\rho_{\vartheta}=3 n^{2} m_{p l}^{2} L^{-2},
$$

where $n, m_{p l}$, and $L$ are the numerical constant, reduced Planck mass, and IR cutoff, respectively. In order to illustrate 
the accelerated expansion of the universe in a better way, different IR cutoffs $(L)$ have been developed.

Moreover, different modified theories of gravity have also been developed for explaining the accelerated expansion. The Chern-Simons modified gravity is one of them which is recently developed [27] and it is not a random extension. However, this modification is motivated from string theory (as a necessary anomaly-canceling term to conserve unitarity [28]) and loop quantum gravity [29]. Also, this modification displays the violation of parity symmetry in Einstein-Hilbert action due to the addition of the Pontryagin density (which is a simply topological term in four dimensions, unless the coupling constant is not constant or promoted to a scalar field). The details of this proposal for a correction to general relativity have been given in [30]. Recently, some people discuss the cosmological scenario/solutions with the inclusion of various HDE models [31-34].

In this paper, we study some DE models such as pilgrim DE (PDE) with Hubble and event horizons in the framework of dynamical Chern-Simons modified gravity. We discuss equation of state (EoS) and Hubble parameters, cosmological plane using EoS parameter, and stability of the models in interacting scenario. We examine these scenarios with respect to redshift function, interacting parameter, and PDE parameter. We check the compatibility of these results by comparing with the data from recent observations. The paper is organized as follows. In the next section, we briefly discuss the dynamics of Chern-Simons modified gravity. Section 3 provides the scenario of dark energy models which involves Hubble and event horizons PDE models. In Section 4, we discuss the cosmological parameters, cosmological plane, and stability scenario for both of these models. Also we give comparison of obtained results with observational data as well as results in literature. Section 5 concludes the discussion.

\section{Dynamical Chern-Simons Modified Gravity}

The action which describes the Chern-Simons theory is defined as follows [30, 35-37]:

$$
\begin{aligned}
S= & \frac{1}{16 \pi G} \int d^{4} x\left[\sqrt{-g} R+\frac{\ell}{4} \theta^{\star} R^{\rho \sigma \mu \nu} R_{\rho \sigma \mu \nu}\right. \\
& \left.-\frac{1}{2} g^{\mu \nu} \nabla_{\mu} \theta \nabla_{\nu} \theta+V(\theta)\right]+S_{\text {mat }} .
\end{aligned}
$$

Here, $R,{ }^{\star} R^{\rho \sigma \mu \nu} R_{\rho \sigma \mu \nu}, \ell, \theta, S_{\text {mat }}$, and $V(\theta)$ are Ricci scalar, a topological invariant called the Pontryagin term, coupling constant, dynamical variable, action of matter, and the potential, respectively. We set $V(\theta)=0$ for simplicity. By varying the above action according to the metric $g_{\mu \nu}$ as well as the scalar field $\theta$, we obtain the following field equations:

$$
\begin{aligned}
G_{\mu \nu}+\ell C_{\mu \nu} & =8 \pi G T_{\mu \nu}, \\
g^{\mu \nu} \nabla_{\mu} \nabla_{\nu} \theta & =-\frac{\ell}{64 \pi}{ }^{\star} R^{\rho \sigma \mu \nu} R_{\rho \sigma \mu \nu}
\end{aligned}
$$

respectively. In these equations, $G_{\mu \nu}$ and $C_{\mu \nu}$ are the Einstein and Cotton tensors, respectively. The Cotton tensor is defined as follows:

$$
\begin{aligned}
C^{\mu \nu}= & -\frac{1}{2 \sqrt{-g}}\left(\left(\nabla_{\rho} \theta\right) \varepsilon^{\rho \beta \tau(\mu} \nabla_{\tau} R_{\beta}^{\nu)}\right) \\
& +\left(\nabla_{\sigma} \nabla_{\rho} \theta\right)^{\star} R^{\rho(\mu \nu) \sigma} .
\end{aligned}
$$

In this framework, the energy-momentum tensors have the following forms:

$$
\begin{aligned}
& \widehat{T}_{\mu \nu}^{\theta}=\nabla_{\mu} \theta \nabla_{\nu} \theta-\frac{1}{2} g_{\mu \nu} \nabla^{\rho} \theta \nabla_{\rho} \theta, \\
& T_{\mu \nu}=(\rho+p) U_{\mu} U_{\nu}+p g_{\mu \nu},
\end{aligned}
$$

where $\widehat{T}_{\mu \nu}^{\theta}$ corresponds to scalar field contribution while $T_{\mu \nu}$ represents the $\mathrm{DE}$ and $\mathrm{CDM}$ contributions. Also, $\rho$ represents the energy density due to DE and CDM, while $p$ represents the pressure due to only DE component. Moreover, $U_{\mu}=$ $(1,0,0,0)$ is the four-velocity. Using (3), (4), and (6), we get the following Friedmann equation for flat universe:

$$
H^{2}=\frac{1}{3}\left(\rho_{c d m}+\rho_{\vartheta}\right)+\frac{1}{6} \dot{\theta}^{2},
$$

where $H=\dot{a} / a$ is the Hubble parameter and the dot denotes the derivative of scale factor $a$ with respect to cosmic time and $m_{p l}^{-1}=8 \pi G=1$.

Field equation (4) is associated with the scalar field and ${ }^{\star} R^{\rho \sigma \mu \nu} R_{\rho \sigma \mu \nu}=0$ for FRW metric. In this scenario, (4) takes the form

$$
g^{\mu \nu} \nabla_{\mu} \nabla_{\nu} \theta=g^{\mu \nu}\left[\partial_{\mu} \partial_{\nu} \theta-\Gamma_{\mu \nu}^{\rho} \partial_{\rho} \theta\right]=0
$$

By assuming $\theta=\theta(t)$, we can obtain the equation

$$
\ddot{\theta}+3 H \dot{\theta}=0,
$$

yielding the solution

$$
\dot{\theta}=b a^{-3}
$$

where $b$ is an integration constant. In this way, (7) takes the form

$$
H^{2}=\frac{1}{3}\left(\rho_{c d m}+\rho_{\vartheta}\right)+\frac{1}{6} b^{2} a^{-6} .
$$

The continuity equation in this framework becomes [31]

$$
\dot{\rho}+3 H(\rho+p)=0 .
$$

Taking the interaction between CDM and DE into account, the continuity equation may be written as

$$
\begin{aligned}
\dot{\rho}_{c d m}+3 H \rho_{c d m} & =\Delta, \\
\dot{\rho}_{\vartheta}+3 H \rho_{\vartheta}\left(1+\omega_{\vartheta}\right) & =-\Delta,
\end{aligned}
$$

where $\Delta$ serves as interaction term between CDM and $\mathrm{DE}$ which has dynamical nature. The ambiguous nature of 
CDM as well as DE creates the problem for the choice of interaction term. It is difficult to describe interaction via the first principle. However, the continuity equation provides a clue about the form of interaction; that is, it must be a function of the product of energy density and a term with units of time (such as Hubble parameter). With this idea, different forms for interaction have been proposed. We take the following form of this interaction term:

$$
\Delta=3 d^{2} H \rho_{c d m}
$$

with $d^{2}$ as an interaction parameter which exchanges the energy between CDM and DE components. By incorporating this $\Delta$ in (13), we get

$$
\rho_{c d m}=\rho_{c d m 0} a^{-3\left(1-d^{2}\right)} .
$$

\section{Dark Energy Models}

The idea of Cohen et al. [26] is reconsidered by Wei [14] with the proposal of PDE. According to Wei, the $\mathrm{BH}$ formation can be avoided through appropriate resistive force which is capable of preventing the matter collapse. In this phenomenon, phantomlike DE can play an important role which possesses strong repulsive force as compared to quintessence DE. The effective role of phantomlike $\mathrm{DE}$ onto the mass of $\mathrm{BH}$ in the universe has also been observed in many different ways. The accretion phenomenon is one of them which favors the possibility of avoidance of $\mathrm{BH}$ formation due to the presence of phantomlike DE in the universe. It has been suggested that accretion of phantom DE (which is attained through family of Chaplygin gas models [38-44]) reduces the mass of $\mathrm{BH}$.

It is strongly believed that the presence of phantom DE in the universe will force it towards big-rip singularity. This represents that the phantomlike universe possesses ability to prevent the $\mathrm{BH}$ formation. The proposal of PDE model [14] also works on this phenomenon which states that phantom DE contains enough repulsive force which can resist against the $\mathrm{BH}$ formation. The energy density of PDE has the following form:

$$
\rho_{\vartheta}=3 n^{2} m_{p}^{4-u} L^{-u}
$$

where both $n$ and $u$ are dimensionless constants. Wei [14] developed cosmological parameters for PDE model with Hubble horizon and provided different possibilities for avoiding the $\mathrm{BH}$ formation through $\mathrm{PDE}$ parameter. The first property of PDE is

$$
\rho_{\vartheta} \gtrsim m_{p}^{2} L^{-2}
$$

From (17) and (18), we have $L^{2-u} \gtrsim m_{p}^{u-2}=l_{p}^{2-u}$, where $l_{p}$ is the reduced Plank length. Since $L>l_{p}$, one requires

$$
u \leq 2
$$

The second requirement for PDE is that it gives phantomlike behavior [14]

$$
\omega_{\vartheta}<-1
$$

It is stated [14] that a particular cutoff $L$ has to choose to obtain the EoS for PDE. For instance, radius of Hubble horizon $L=H^{-1}$, event horizon $L=R_{E}=a \int_{t}^{\infty}(d t / a)$, and the form $L=\left(H^{2}+\dot{H}\right)^{-1 / 2}$ represented the Ricci length, the Granda-Oliveros length $\left(\alpha H^{2}+\beta \dot{H}\right)^{-1 / 2}[5]$, and so forth.

In the present work, we choose Hubble as well as event horizons for the cosmological analysis taking PDE model in the underlying gravity.

(i) Hubble Horizon PDE. The Hubble horizon is the pioneer horizon which is used as an IR cutoff or length scale for HDE model. In the beginning, HDE with this horizon has suffered a problem that its EoS parameter does not give consistent behavior with present day observations about the universe [12]. This shortcoming has been settled down with the passage of time and suggested that HDE with Hubble horizon possesses the ability to explain current scenario of the universe in the presence of interaction with DM $[45,46]$. Moreover, the results of various cosmological parameters in the scenario of HDE model with Hubble scale have been investigated through different observational data [47, 48]. Recently, some authors $[15,49]$ have evaluated this model by taking interaction with CDM and pointed out that it can explain the present scenario of the universe. Here, we use PDE with this horizon and find different cosmological parameters. Thus the energy density of PDE model with Hubble horizon is given as follows:

$$
\rho_{\vartheta}=3 n^{2} m_{p}^{4-u} H^{u}
$$

(ii) Event Horizon PDE. Li [12] proposed event horizon as an IR cutoff and argued that its EoS parameter corresponds to the DE era of the universe. He found that HDE parameter plays a crucial role to obtain desired results of present eras of the universe. Later on, many discussions about cosmic acceleration have been made by choosing this HDE model which provides different constraints on EoS parameter [50-53]. The validity of thermodynamics laws has also been discussed by taking event horizon as a boundary of cosmological system [52-57]. In addition, different cosmological schemes have been used to check the viability of HDE with event horizon [58-61]. The event horizon is given as

$$
\begin{aligned}
& L=a(t) \int_{t}^{\infty} \frac{d \tilde{t}}{a(\tilde{t})}, \\
& \dot{L}=H L-1 .
\end{aligned}
$$

\section{Cosmological Analysis}

In this section, we study the cosmological parameters such as EoS and Hubble parameters and plane which involves $\omega_{\vartheta}-\omega_{9}^{\prime}$ plane and check the stability criteria for both Hubble horizon PDE and event horizon PDE in dynamical Chern-Simons gravity.

4.1. Cosmological Parameters. Here, we address the discussion of the basic cosmological parameters such as Hubble and 
EoS for PDE models. Firstly we require the rate of change of PDE with Hubble horizon which is given by

$$
\dot{\rho}_{\vartheta}=u \rho_{\vartheta} \frac{\dot{H}}{H}
$$

By taking the differentiation of (11) and using (16), (23) with $a=a_{0}(1+z)^{-1}$, we extract the expression of Hubble parameter for analyzing its behavior as follows:

$$
\frac{\dot{H}}{H^{2}}=\frac{\left(3\left(d^{2}-1\right) H_{0}^{2} \Omega_{m 0} a^{-3\left(1-d^{2}\right)}-b^{2} a^{-6}\right)}{2 H^{2}-u n^{2} m_{p}^{2-u} H^{u}} .
$$

The numerical display of the above differential equation for $H$ versus $1+z$ is shown in Figure 1 for various values of $u$ and $d^{2}$. The other constant parameters are $H_{0}=74, \Omega_{m 0}=0.23, n=$ 0.91 , and $b=2$. For $u=2$ (upper left panel), we can observe that the evolution of Hubble parameter lies within the range $75 \pm 0.5$ for all values of interacting parameter. However, it lies in the range $(74,74.30)$ for all $d^{2}$ as well as for $u=1,-1,-2$ as shown in the right upper and lower panels.
The EoS parameter can be obtained with the help of (11), (16), (23), and (24) as follows:

$$
\begin{aligned}
\omega_{\vartheta}= & -1-d^{2} \frac{H_{0}^{2} \Omega_{m 0} a^{-3\left(1-d^{2}\right)}}{n^{2} m_{p}^{4-u} H^{u}} \\
& +\frac{u}{3}\left[\frac{3\left(d^{2}-1\right) H_{0}^{2} \Omega_{m 0} a^{-3\left(1-d^{2}\right)}-b^{2} a^{-6}}{2 H^{2}-u n^{2} m_{p}^{2-u} H^{u}}\right] .
\end{aligned}
$$

The plots of $\omega_{\vartheta}$ versus $1+z$ for the same constant cosmological parameters are shown in Figure 2. It can be observed that EoS parameter exhibits the phantomlike behavior for all cases of $u$ and $d^{2}$, except for the case of $u=1$ (where EoS shows variation from vacuum towards quintessence regions). Moreover, the EoS parameter lies in the ranges $-1.24 \leq \omega_{\vartheta} \leq$ -1 (for $u=2),-1 \leq \omega_{\vartheta} \leq-0.2$ (for $\left.u=1\right),-1.30 \leq \omega_{\vartheta} \leq-1$ (for $u=-1$ ), and $-2.30 \leq \omega_{\vartheta} \leq-1$ (for $u=-2$ ).

The time derivative of event horizon PDE takes the form

$$
\dot{\rho}_{\vartheta}=-u \rho_{\vartheta}\left(H-L^{-1}\right) \text {. }
$$

The evolutionary form of Hubble parameter of event horizon PDE turns out to be

$$
\begin{aligned}
& \frac{d H}{d z}=(2(1+z) H)^{-1}\left(3\left(1-d^{2}\right) \Omega_{m 0} H_{0}^{2}(1+z)^{-3\left(d^{2}-1\right)}+b^{2}(1+z)^{6}\right. \\
& \quad+n^{2} u\left(1-H^{-1}\left(n^{-2}\left(H^{2}-\Omega_{m 0} H_{0}^{2}(1+z)^{-3\left(d^{2}-1\right)}-6^{-1}\left(b^{2}(1+z)^{6}\right)\right)\right)^{1 / u}\right)\left(n ^ { - 2 } \left(H^{2}-\Omega_{m 0} H_{0}^{2}(1+z)^{-3\left(d^{2}-1\right)}\right.\right. \\
& \left.\left.\left.\quad-6^{-1} b^{2}(1+z)^{6}\right)\right)\right)
\end{aligned}
$$

Its numerical display versus $1+z$ for three different values of $d^{2}$ is shown in Figure 3. For $u=2$ (upper left panel), Hubble parameter remains in the range $74.80_{-0.005}^{+0.005}$. Moreover, the Hubble parameter increases with the passage of time for the cases $u=1,-1$. However, it lies between 73.4 and 74 for the case $u=-2$ (lower right panel).

For the event horizon, the EoS parameter turns out to be

$$
\begin{aligned}
\omega_{\vartheta}= & -1-\left(d^{2} \Omega_{m 0} H_{0}^{2}(1+z)^{-3\left(d^{2}-1\right)}\left(\left(H^{2}-\Omega_{m 0} H_{0}^{2}(1+z)^{-3\left(d^{2}-1\right)}-6^{-1} b^{2}(1+z)^{6}\right)\right)^{-1}\right) \\
& -9^{-1}\left(u\left(1-H^{-1}\left(n^{-2}\left(H^{2}-\Omega_{m 0} H_{0}^{2}(1+z)^{-3\left(d^{2}-1\right)}-6^{-1} b^{2}(1+z)^{6}\right)\right)^{1 / u}\right)\right) .
\end{aligned}
$$

We analyze the behavior of above EoS parameter versus redshift parameter through PDE as well as interacting parameters (Figure 4$)$. The present values of EoS parameter are $\omega_{90}=-0.98$ (for $\left.u=2\right), \omega_{90}=-1$ (for $\left.u=1\right), \omega_{90}=-0.88$ (for $u=-1$ ), and $\omega_{90}=-0.98$ (for $u=-0.78$ ). We also observe that all the trajectories of EoS parameter cross the phantom divide line from quintessence region and go towards phantom region in all the cases of $u=2,-1,-2$ except for $u=1$ case (where it starts from $\Lambda$ CDM limit and goes towards phantom era). However, the ranges of EoS parameter lie within the ranges of observational constraints.

4.2. Cosmological Plane. Taking the evolutionary form of EoS parameter as $\omega_{\vartheta}^{\prime}$, the $\omega_{\vartheta}-\omega_{\vartheta}^{\prime}$ plane is used to explore the dynamical property of DE models where prime shows evolution with respect to $\ln a$. Caldwell and Linder [62] were the first who introduced this method for studying the behavior of quintessence scalar field DE model. Using 

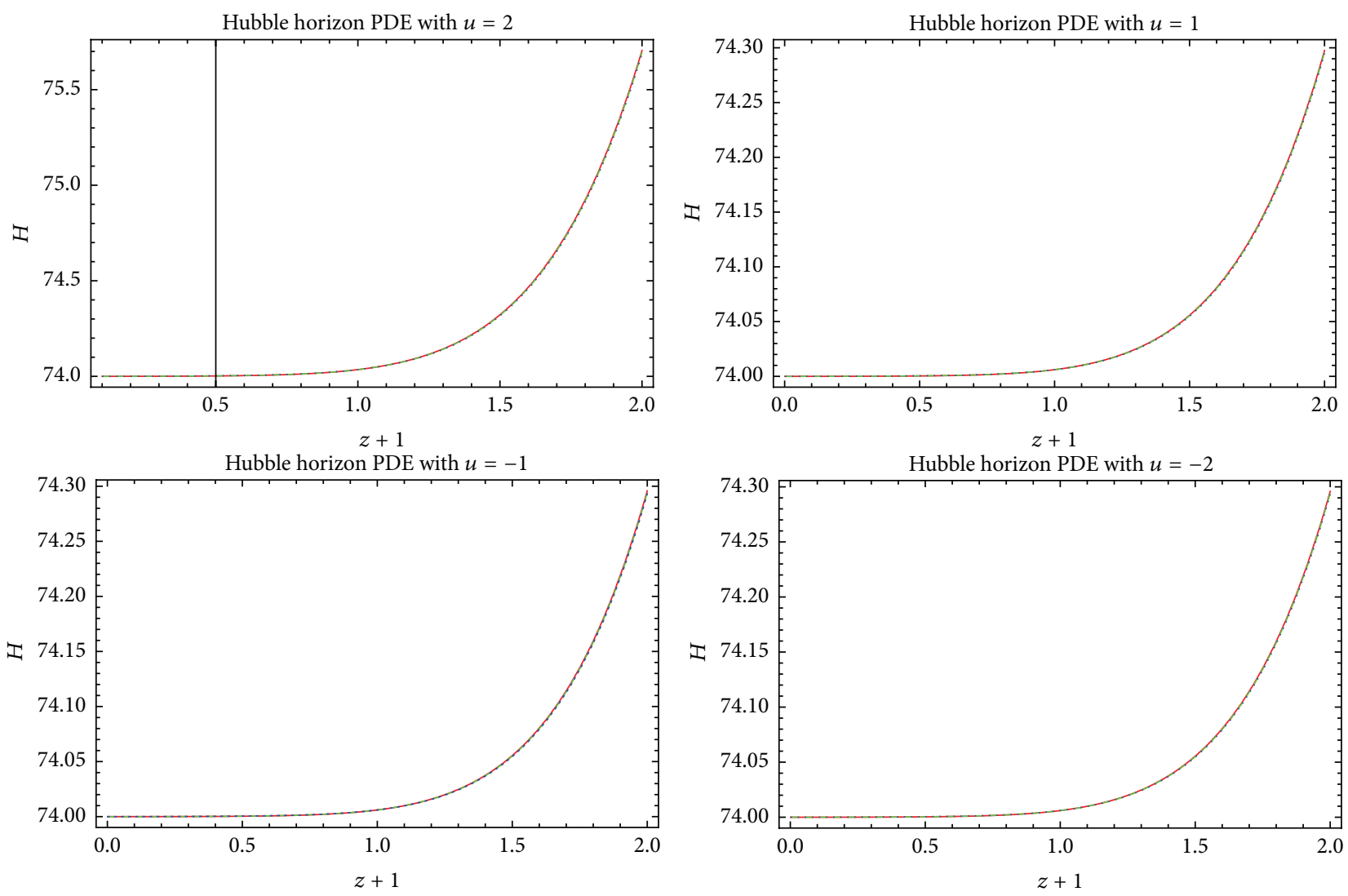

$$
\begin{aligned}
-d^{2} & =0.2 \\
---d^{2} & =0.3 \\
\cdots \cdots & d^{2}=0.4
\end{aligned}
$$

$$
\begin{aligned}
- & d^{2}=0.2 \\
---d^{2} & =0.3 \\
\cdots \cdots & d^{2}=0.4
\end{aligned}
$$

Figure 1: Plots of $H$ versus $1+z$ for Hubble horizon PDE model in dynamical Chern-Simons gravity.

the obtained results, they divided the $\omega_{\vartheta}-\omega_{\vartheta}^{\prime}$ plane into two categories:

(i) Thawing region is the region where EoS parameter nearly evolves from -1 and increases with time while its evolution parameter expresses positive behavior; that is, $\omega_{\vartheta}^{\prime}>0$ for $\omega_{\vartheta}<0$.

(ii) In freezing region, the evolution parameter for EoS parameter remains negative; that is, $\omega_{\vartheta}^{\prime}<0$ for $\omega_{\vartheta}<0$.

Taking derivative of (25) with respect to $x=\ln a$ and using (24), we get $\omega_{9}^{\prime}$ for Hubble horizon PDE as follows:

$$
\begin{aligned}
& \omega_{\vartheta}^{\prime}=3^{-1}\left(\left(9\left(-1+d^{2}\right) d^{2} \Omega_{m 0} H_{0}^{2}(1+z)^{2-3 d^{2}} H^{-u}\right)\right. \\
& \cdot n^{-2}+\left(-6 b^{2} u(1+z)^{5}-9\left(-1+d^{2}\right)^{2}\right. \\
& \left.\cdot \Omega_{c d m 0} H_{0}^{2} u(1+z)^{2-3 d^{2}}\right)\left(-2 H^{2}+3 n^{2} u H^{u}\right)^{-1} \\
& +\left(u(1+z)^{5-6 d^{2}}\right.
\end{aligned}
$$

$$
\begin{aligned}
& \cdot\left(-3\left(-1+d^{2}\right) \Omega_{c d m 0} H_{0}^{2}+b^{2}(1+z)^{3+3 d^{2}}\right)^{2} \\
& \left.\cdot\left(4 H^{2}-3 n^{2} u^{2} H^{u}\right)\right)\left(\left(2 H^{2}-3 n^{2} u H^{u}\right)^{2}\right. \\
& \left.\cdot\left(-2 H^{2}+n^{2} u H^{u}\right)\right)^{-1} \\
& -\left(3 d^{2} \Omega_{c d m 0} H_{0}^{2} u(1+z)^{5-6 d^{2}}\right. \\
& \left.\cdot\left(-3\left(-1+d^{2}\right) \Omega_{c d m 0} H_{0}^{2}+b^{2}(1+z)^{3+3 d^{2}}\right)\right) \\
& \left.\cdot\left(n^{4} u H^{2 u}-2 n^{2} H^{2+u}\right)^{-1}\right) .
\end{aligned}
$$

For event horizon PDE, $\omega_{9}^{\prime}$ takes the form

$$
\begin{aligned}
\omega_{\vartheta}^{\prime} & =\left(-648 d^{2} \Omega_{m 0} H_{0}^{2}(1+z)^{3} H^{3}\left(\left(-3+3 d^{2}+u\right) H\right.\right. \\
& -u\left(\left(-6^{-1} b^{2}(1+z)^{6}-\Omega_{m 0} H_{0}^{2}(1+z)^{3-3 d^{2}}+H^{2}\right)\right.
\end{aligned}
$$



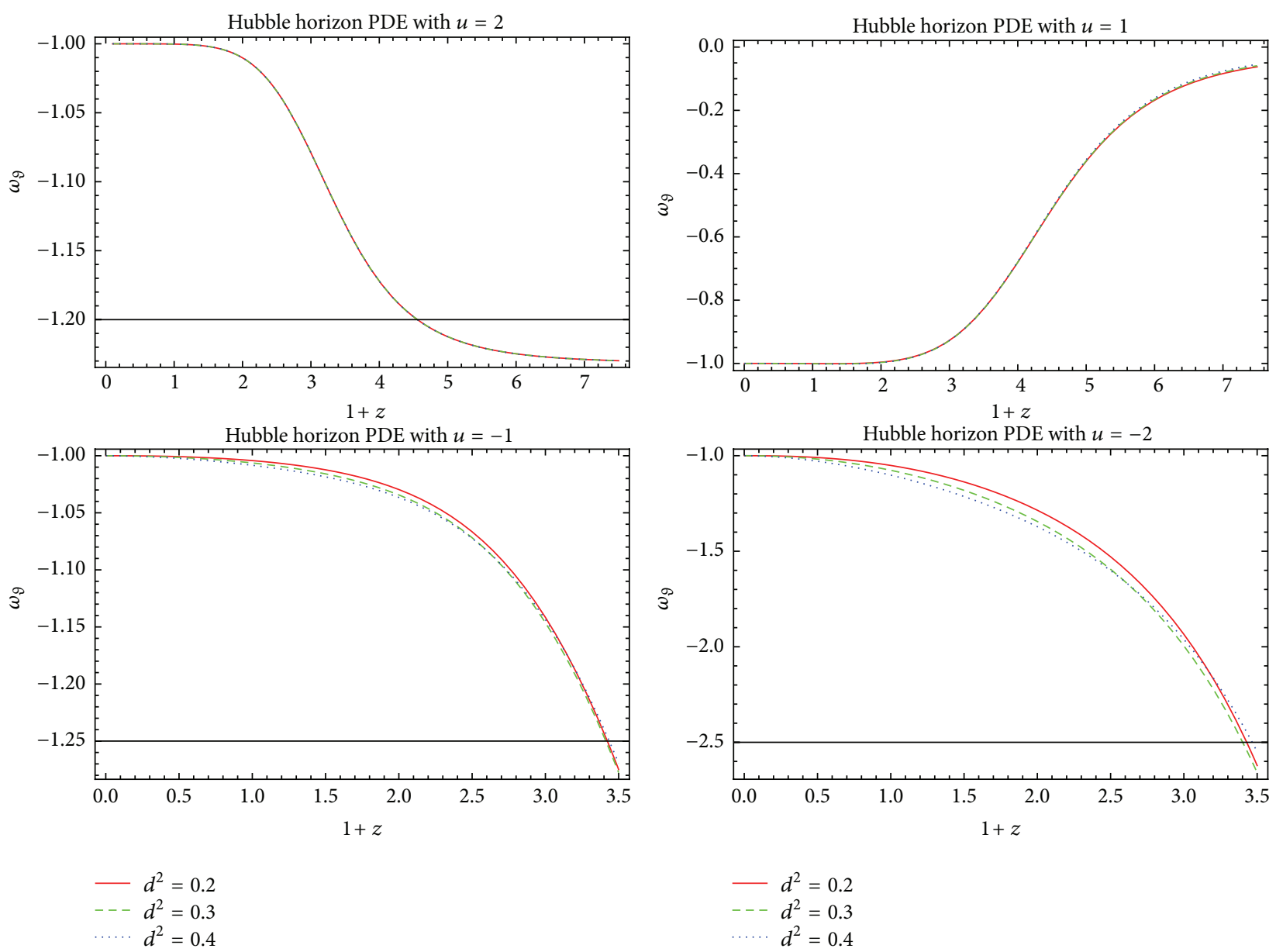

FIgure 2: Plots of $\omega_{\vartheta}$ versus $1+z$ for Hubble horizon PDE model in dynamical Chern-Simons gravity with $u=2,1,-1,-2$.

$$
\begin{aligned}
& \left.\left.\left.\cdot n^{-2}\right)^{1 / u}\right)+6^{-1 / u}\right) u(1+z)^{-3 d^{2}}\left(-\left(\left(b^{2}(1+z)^{6}\right.\right.\right. \\
& \left.\left.\left.+6 \Omega_{m 0} H_{0}^{2}(1+z)^{3-3 d^{2}}-6 H^{2}\right) n^{-2}\right)\right)^{1 / u}\left(6 \Omega_{m 0} H_{0}^{2}(1+z)^{3}\right. \\
& \left.+(1+z)^{3 d^{2}}\left(b^{2}(1+z)^{6}-6 H^{2}\right)\right)\left(H \left(6 \Omega _ { m 0 } H _ { 0 } ^ { 2 } \left(-3+3 d^{2}\right.\right.\right. \\
& +u)(1+z)^{3}+(1+z)^{3 d^{2}}\left(b^{2}(-6+u)(1+z)^{6}-6(-2+u)\right. \\
& \left.\left.\cdot H^{2}\right)\right) \\
& -6^{-1 / u}\left(-\left(\left(b^{2}(1+z)^{6}+6 \Omega_{m 0} H_{0}^{2}(1+z)^{3-3 d^{2}}-6 H^{2}\right)\right.\right. \\
& \left.\left.\cdot n^{-2}\right)\right)^{1 / u}\left(6 \Omega_{m 0} H_{0}^{2} u(1+z)^{3}+(1+z)^{3 d^{2}}\left(b^{2} u(1+z)^{6}\right.\right. \\
& \left.\left.\left.-6(-2+u) H^{2}\right)\right)\right)\left(1 0 8 ( 1 + z ) H ^ { 4 } \left(6 \Omega_{m 0} H_{0}^{2}(1+z)^{3}+(1\right.\right. \\
& \left.\left.+z)^{3 d^{2}}\left(b^{2}(1+z)^{6}-6 H^{2}\right)\right)\right)^{-1} \cdot
\end{aligned}
$$

The $\omega_{9}-\omega_{9}^{\prime}$ plane for Hubble as well as event horizons PDE with different values of $u$ has been displayed in Figures 5 and 6, respectively. For Hubble horizon PDE (Figure 5), the $\omega_{9}-\omega_{\vartheta}^{\prime}$ plane meets the $\Lambda$ CDM model, that is, $\left(\omega_{\vartheta}, \omega_{\vartheta}^{\prime}\right)=(-1,0)$, in all cases of $d^{2}$ for $u=1$ (right upper panel) and $u=-1$ (left lower panel). Also, the trajectories satisfy the thawing region (for $u=2,-1,-2$ ) and freezing region (for $u=1$ ). In case of event horizon PDE (Figure 6), the $\omega_{\vartheta}-\omega_{9}^{\prime}$ plane meets the $\Lambda$ CDM model for $u=-1$ only. Moreover, the trajectories meet the freezing region for all cases of $u$ and $d^{2}$.

4.3. The Stability Analysis. The stability analysis of PDE models in the present framework is being discussed in this section. For this purpose, we extract the following squared speed of sound expression:

$$
v_{s}^{2}=\frac{\dot{p}_{\vartheta}}{\dot{\rho}_{\vartheta}}=\frac{p_{\vartheta}^{\prime}}{\rho_{\vartheta}^{\prime}} .
$$

Inserting corresponding expressions and after some calculations, we obtain squared speed of sound as follows: 

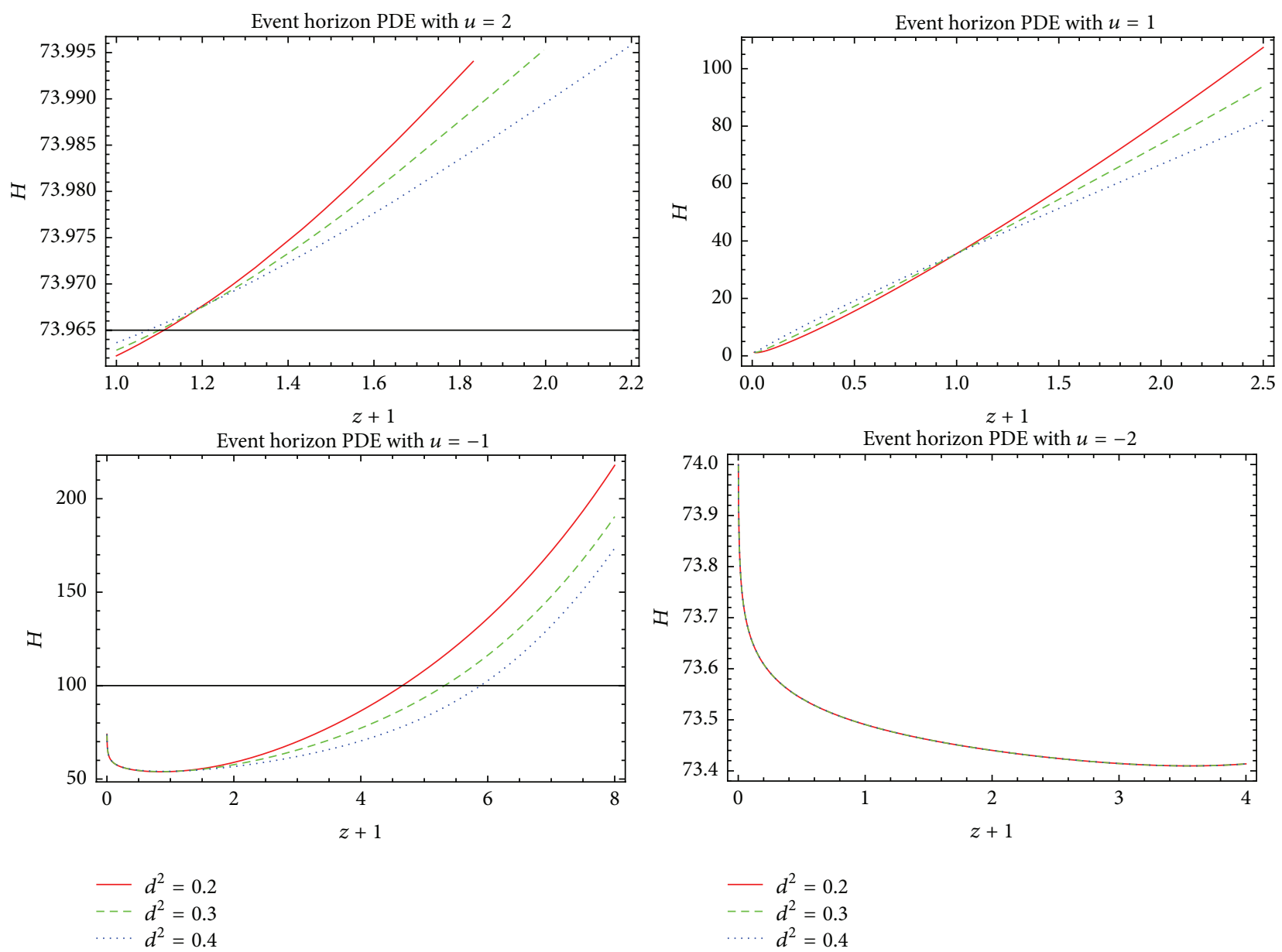

FIGURE 3: Plots of $H$ versus $1+z$ for event horizon PDE model in dynamical Chern-Simons gravity.

$$
\begin{aligned}
v_{s}^{2} & =3^{-1}\left(-3-\left(3 \Omega_{m 0}^{2} H_{0}^{2}(1+z)^{3-3 d^{2}} H^{-u}\right) n^{-2}+\left(-b^{2} u(1+z)^{6}+3\left(-1+d^{2}\right) \Omega_{m 0} H_{0}^{2} u(1+z)^{3-3 d^{2}}\right)\left(-2 H^{2}\right.\right. \\
& \left.+3 n^{2} u H^{u}\right)^{-1}+\left(( 2 H ^ { 2 } - 3 n ^ { 2 } u H ^ { u } ) \left(\left(9\left(-1+d^{2}\right) d^{2} \Omega_{m 0} H_{0}^{2}(1+z)^{2-3 d^{2}} H^{-u}\right) n^{-2}\right.\right. \\
& +\left(-6 b^{2} u(1+z)^{5}-9\left(-1+d^{2}\right)^{2} \Omega_{m 0} H_{0}^{2} u(1+z)^{2-3 d^{2}}\right)\left(-2 H^{2}+3 n^{2} u H^{u}\right)^{-1} \\
& +\left(u(1+z)^{5-6 d^{2}}\left(-3\left(-1+d^{2}\right) \Omega_{m 0} H_{0}^{2}+b^{2}(1+z)^{3+3 d^{2}}\right)^{2}\left(4 H^{2}-3 n^{2} u^{2} H^{u}\right)\right) \\
& \cdot\left(\left(2 H^{2}-3 n^{2} u H^{u}\right)^{2}\left(-2 H^{2}+n^{2} u H^{u}\right)\right)^{-1}-\left(3 d^{2} \Omega_{m 0} H_{0}^{2} u(1+z)^{5-6 d^{2}}\left(-3\left(-1+d^{2}\right) \Omega_{m 0} H_{0}^{2}+b^{2}(1+z)^{3+3 d^{2}}\right)\right) \\
& \left.\left.\left.\cdot\left(n^{4} u H^{2 u}-2 n^{2} H^{2+u}\right)^{-1}\right)\right)\left(-b^{2}(1+z)^{6}+3\left(-1+d^{2}\right) \Omega_{m 0} H_{0}^{2}(1+z)^{3-3 d^{2}}\right)^{-1}\right) .
\end{aligned}
$$

The squared speed of sound is plotted against redshift parameter in Figure 7 with the same constant parameters. We can observe from the plot (for $u=2$ ) that the squared speed of sound remains positive for the present as well as later epochs exhibiting stability of the model. For the other case $u$, squared speed of sound exhibits the instability at the present epoch while it shows stability of the models for the later epoch. 

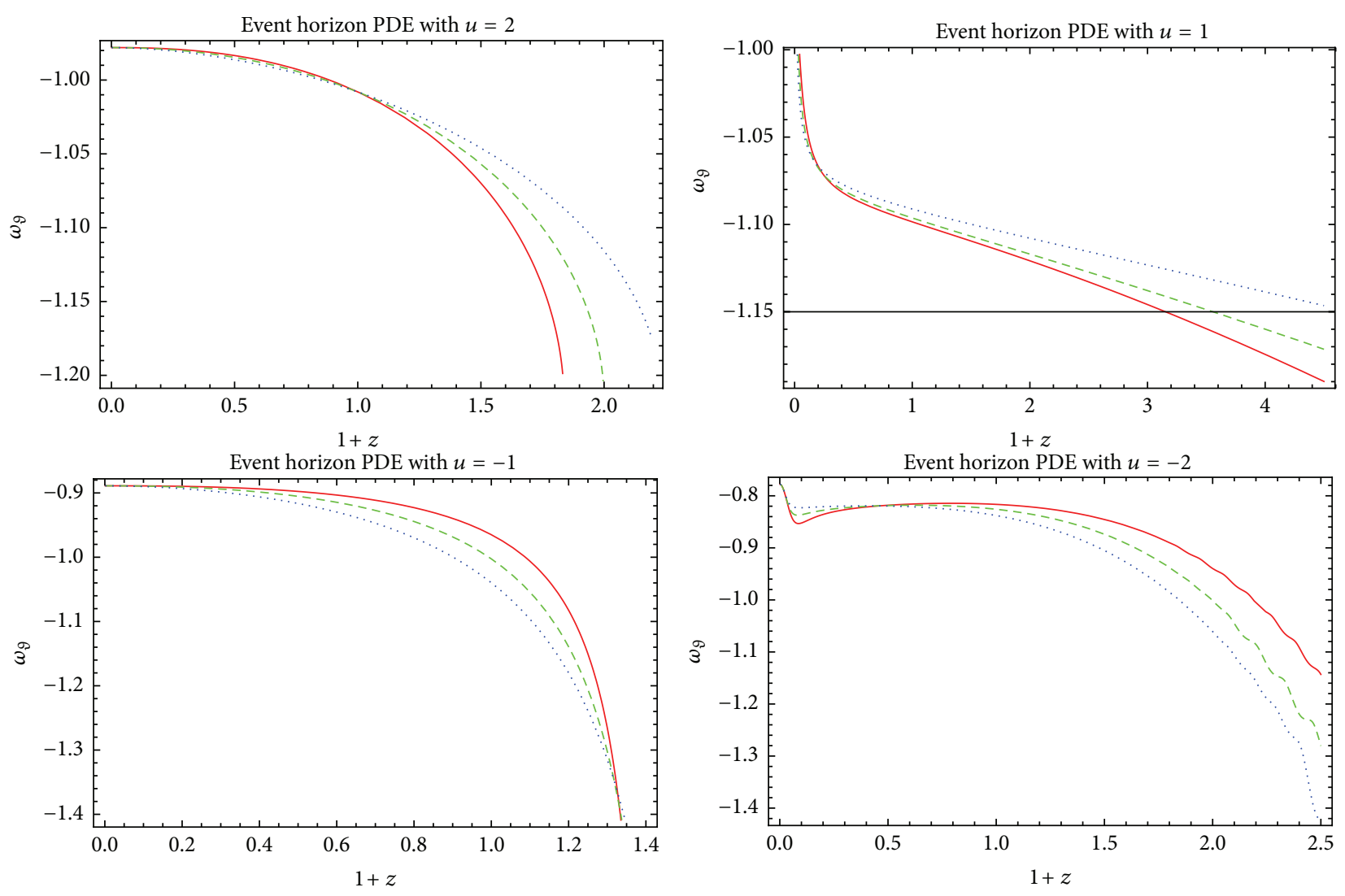

$$
\begin{aligned}
-d^{2} & =0.2 \\
---d^{2} & =0.3 \\
\cdots \cdots d^{2} & =0.4
\end{aligned}
$$

$$
\begin{aligned}
-d^{2} & =0.2 \\
--d^{2} & =0.3 \\
\cdots \cdot d^{2} & =0.4
\end{aligned}
$$

FIgURE 4: Plots of $\omega_{\vartheta}$ versus $1+z$ for Hubble horizon PDE model in dynamical Chern-Simons gravity with $u=2,1,-1,-2$.

For event horizon PDE, the squared speed of sound becomes

$$
\begin{aligned}
v_{s}^{2} & =-1+6 d^{2} \Omega_{m 0} H_{0}^{2}(1+z)^{3}\left(6 \Omega_{m 0} H_{0}^{2}(1+z)^{3}+(1+z)^{3 d^{2}}\left(b^{2}(1+z)^{6}-6 H^{2}\right)\right)^{-1}+(u(-H \\
& \left.\left.+\left(\left(-6^{-1} b^{2}(1+z)^{6}-\Omega_{m 0} H_{0}^{2}(1+z)^{3-3 d^{2}}+H^{2}\right) n^{-2}\right)^{1 / u}\right)\right)(9 H)^{-1}+648 d^{2} \Omega_{m 0} H_{0}^{2}(1+z)^{3} H^{3}\left(\left(-3+3 d^{2}+u\right) H\right. \\
& \left.-u\left(\left(-6^{-1} b^{2}(1+z)^{6}-\Omega_{m 0} H_{0}^{2}(1+z)^{3-3 d^{2}}+H^{2}\right) n^{-2}\right)^{1 / u}\right)+6^{1 / u} u(1+z)^{-3 d^{2}} \\
& \cdot\left(-\left(\left(b^{2}(1+z)^{6}+6 \Omega_{m 0} H_{0}^{2}(1+z)^{3-3 d^{2}}-6 H^{2}\right) n^{-2}\right)^{1 / u}\left(6 \Omega_{m 0} H_{0}^{2}(1+z)^{3}+(1+z)^{3 d^{2}}\left(b^{2}(1+z)^{6}-6 H^{2}\right)\right)\right. \\
& \cdot\left(H\left(6 \Omega_{m 0} H_{0}^{2}\left(-3+3 d^{2}+u\right)(1+z)^{3}+(1+z)^{3 d^{2}}\left(b^{2}(-6+u)(1+z)^{6}-6(-2+u) H^{2}\right)\right)\right. \\
& -6^{-1 / u}\left(-\left(\left(b^{2}(1+z)^{6}+6 \Omega_{m 0} H_{0}^{2}(1+z)^{3-3 d^{2}}-6 H^{2}\right) n^{-2}\right)\right)^{1 / u} \\
& \left.\left.\cdot\left(6 \Omega_{m 0} H_{0}^{2} u(1+z)^{3}+(1+z)^{3 d^{2}}\left(b^{2} u(1+z)^{6}-6(-2+u) H^{2}\right)\right)\right)\right)\left(1 0 8 u ( 1 + z ) ^ { 2 } H ^ { 4 } \left(6 \Omega_{m 0} H_{0}^{2}(1+z)^{3}\right.\right. \\
& \left.\left.+(1+z)^{3 d^{2}}\left(b^{2}(1+z)^{6}-6 H^{2}\right)\right)\left(1-\left(\left(-6^{-1} b^{2}(1+z)^{6}-\Omega_{m 0} H_{0}^{2}(1+z)^{3-3 d^{2}}+H^{2}\right) n^{-2}\right)^{1 / u} H^{-1}\right)\right)^{-1} .
\end{aligned}
$$



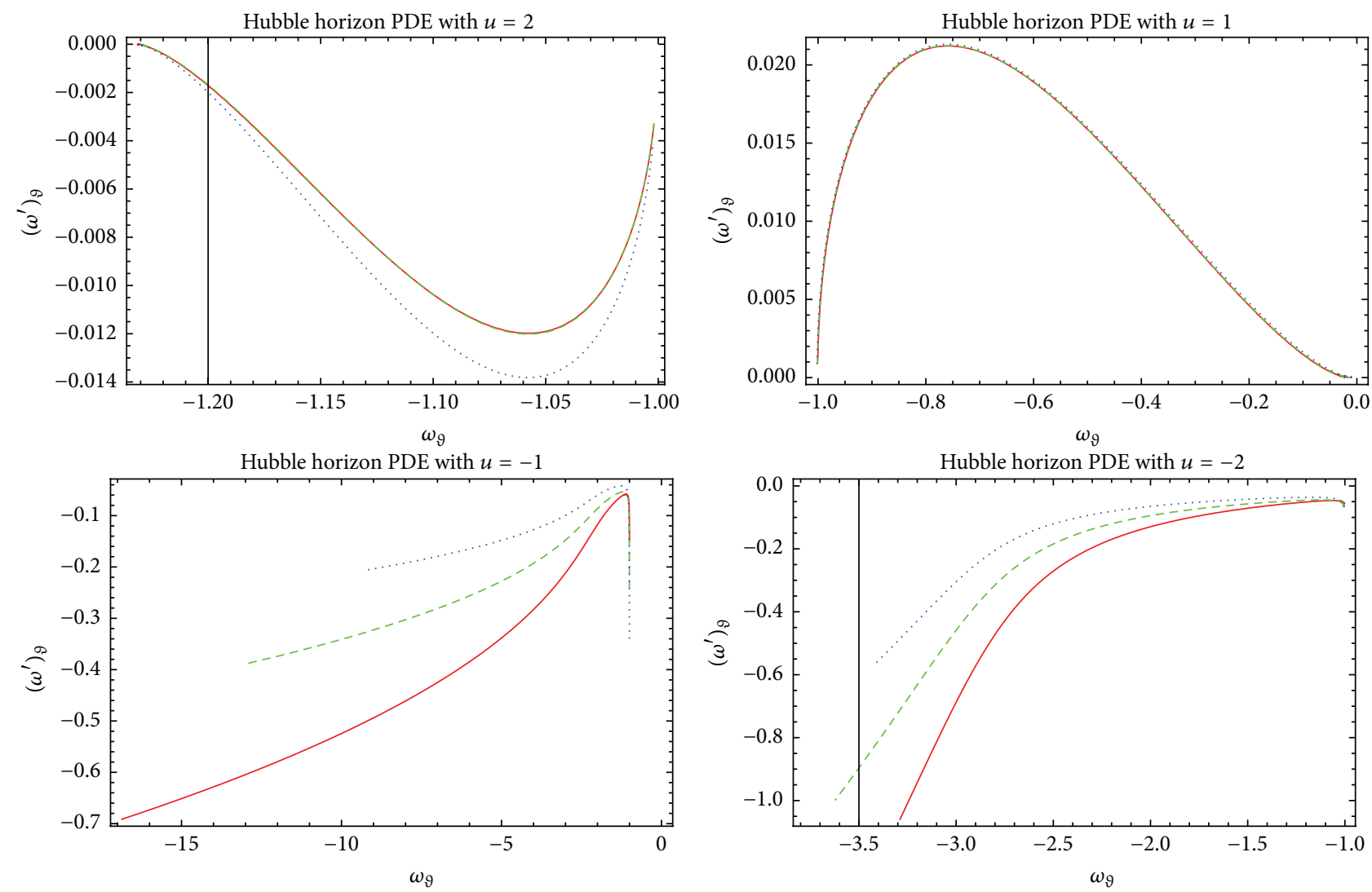

Figure 5: Plots of $\omega_{\vartheta}-\omega_{\vartheta}^{\prime}$ for Hubble horizon PDE model in dynamical Chern-Simons gravity with $u=2,1,-1,-2$.

The plots of this equation are shown in Figure 8. Notice that it exhibits the viability as well as stability of the interacting PDE with event horizon in the present scenario throughout its history.

4.4. Comparison with Observational Data and Literature. Riess et al. [63] found the following ranges of Hubble parameter:

$$
H_{0}=73.8 \pm 2.4 \mathrm{kms}^{-1} \mathrm{Mpc}^{-1},
$$

(Cepheids + SNe Ia)

by using HST observations of Cepheid variables in the host galaxies of eight $\mathrm{SNe}$ of type Ia to calibrate the supernova magnitude-redshift relation within $1 \sigma$ level. Freedman et al. [64] suggested the following best fit constraints:

$$
H_{0}=[74.3 \pm 1.5(\text { statistical }) \pm 2.1(\text { systematic })] \mathrm{kms}^{-1} \mathrm{Mpc}^{-1},
$$

(Carnegie HP).
According to nine-year WMAP observational data [7], Hinshaw et al. provide the following constraints:

$$
\begin{gathered}
H_{0}=72.3 \pm 2.0 \mathrm{kms}^{-1} \mathrm{Mpc}^{-1}, \\
\left(\mathrm{WMAP}+\mathrm{eCMB}+\mathrm{BAO}+H_{0}\right), \\
H_{0}=71.0 \pm 1.3 \mathrm{kms}^{-1} \mathrm{Mpc}^{-1}, \\
\left(\mathrm{WMAP}+\mathrm{eCMB}+\mathrm{BAO}+H_{0}+\mathrm{SNe}\right) .
\end{gathered}
$$

Here, we pointed out that our results of Hubble parameter show consistency with all the above constraints.

It is interesting to mention here that the ranges of EoS parameter for both PDE models lie within the following observational constraints. Ade et al. [65] (Planck data) provided the following constraints for EoS:

$$
\begin{aligned}
& \omega_{\vartheta}=-1.13_{-0.25}^{+0.24}, \quad(\text { Planck }+\mathrm{WP}+\mathrm{BAO}), \\
& \omega_{\vartheta}=-1.09 \pm 0.17, \quad(\text { Planck }+\mathrm{WP}+\text { Union } 2.1), \\
& \omega_{\vartheta}=-1.13_{-0.14}^{+0.13}, \quad(\text { Planck }+\mathrm{WP}+\mathrm{SNLS}), \\
& \omega_{\vartheta}=-1.24_{-0.19}^{+0.18}, \quad\left(\text { Planck }+\mathrm{WP}+H_{0}\right),
\end{aligned}
$$



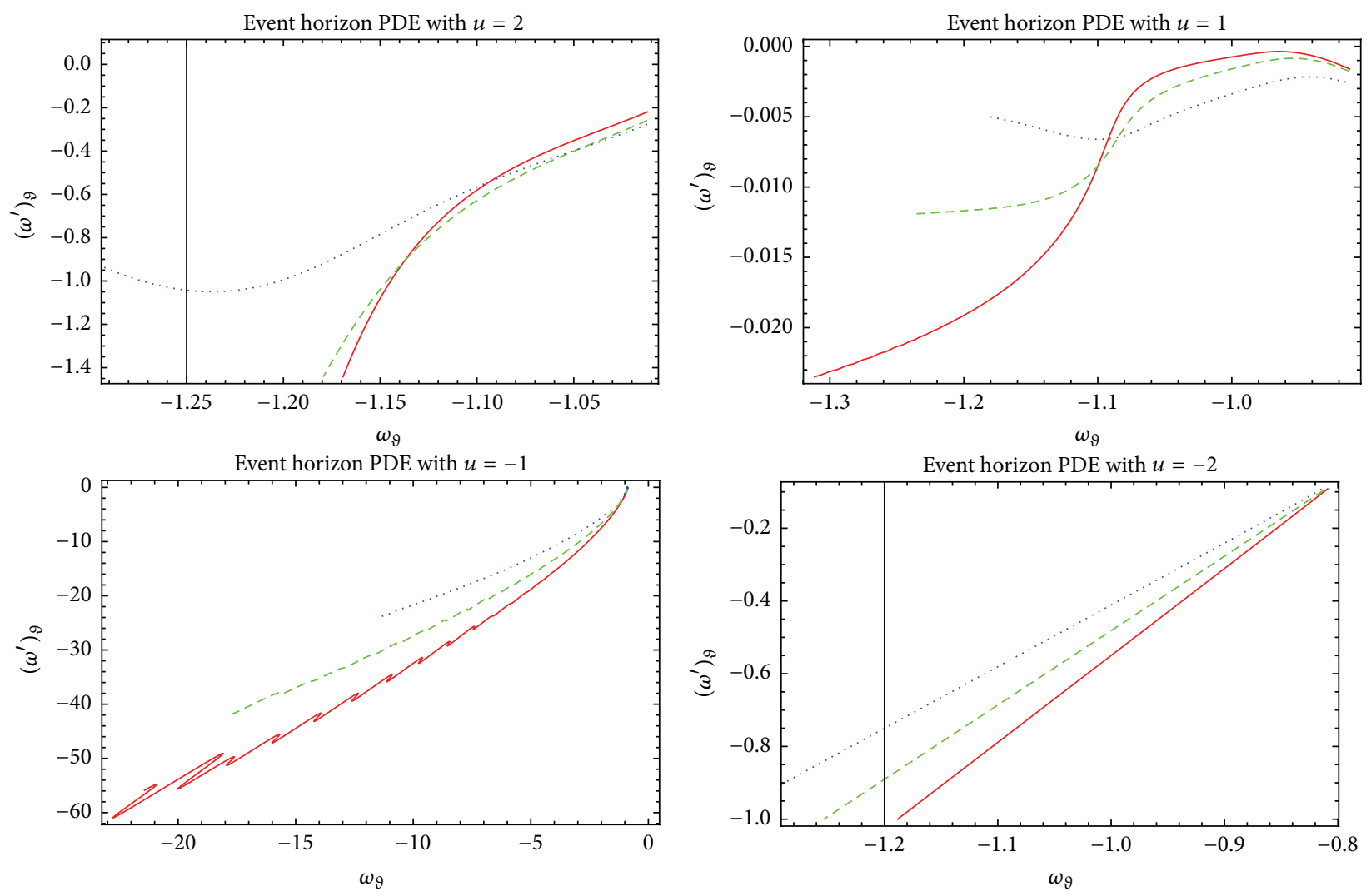

Figure 6: Plots of $\omega_{\vartheta}-\omega_{\vartheta}^{\prime}$ for event horizon PDE model in dynamical Chern-Simons gravity with $u=2,1,-1,-2$.

by implying different combination of observational schemes at $95 \%$ confidence level. The trajectories of EoS parameter also favor the following nine-year WMAP observational data:

$$
\begin{aligned}
& \omega_{\vartheta}=-1.073_{-0.089}^{+0.090}, \\
& \left(\mathrm{WMAP}+\mathrm{eCMB}+\mathrm{BAO}+H_{0}\right), \\
& \omega_{\vartheta}=-1.084 \pm 0.063, \\
& \left(\mathrm{WMAP}+\mathrm{eCMB}+\mathrm{BAO}+H_{0}+\mathrm{SNe}\right) .
\end{aligned}
$$

The constructed planes in both PDE models (Figures 5 and 6) also meet the following constraints as developed by Ade et al. [65]:

$$
\begin{aligned}
& \omega_{\vartheta}=-1.13_{-0.25}^{+0.24}, \quad(\text { Planck }+\mathrm{WP}+\mathrm{BAO}), \\
& \omega_{\vartheta}^{\prime}<1.32, \quad(\text { Planck }+\mathrm{WP}+\mathrm{BAO})
\end{aligned}
$$

at $95 \%$ confidence level. The WMAP nine-year observational data constrain these parameters as follows: using WMAP + $\mathrm{eCAMB}+\mathrm{BAO}+\mathrm{H}_{0}$ data

$$
\begin{aligned}
& \omega_{9}=-1.34 \pm 0.18, \\
& \omega_{9}^{\prime}=0.85 \pm 0.47,
\end{aligned}
$$

and $\mathrm{WMAP}+\mathrm{eCAMB}+\mathrm{BAO}+\mathrm{H}_{0}+\mathrm{SNe}$ data gives

$$
\begin{aligned}
& \omega_{\vartheta}=-1.17_{-0.12}^{+0.13}, \\
& \omega_{\vartheta}^{\prime}=0.85_{-0.49}^{+0.50} .
\end{aligned}
$$

Sharif and Jawad [15] discussed interacting PDE taking three cutoffs (Hubbl horizon, event horizon and conformal age of the universe) in flat FRW universe. It is found that phantom DE is obtained for PDE parameter $u<0$ in all cases except for noninteracting case while it is obtained for different ranges of $u>0$. It is remarked that $u<0$ gives more useful and compatible description for making consensus on the idea of PDE. Taking $\omega_{9}-\omega_{9}^{\prime}$ plane, it is found that $\Lambda \mathrm{CDM}$ is achieved in noninteracting case for PDE with Hubble horizon and conformal age of the universe, while it is achieved for all the cases for PDE with event horizon. In Chern-Simons gravity, the cosmological evolution of the universe is discussed taking Ricci dark energy model with the help of EoS parameter [33]. In this paper, the Eos parameter describes the evolution from stiff matter-dominated universe to DE-dominated universe driven by cosmological constant $(\omega=-1)$. The proposal of PDE is based on the idea that phantom DE possesses enough resistive force to preclude $\mathrm{BH}$ formation. Hence, in the framework of Chern-Simons gravity with help of PDE model, we determine the phantom behavior of the universe.

In another paper [18] taking generalized ghost version of $\mathrm{PDE}$, it is found that the presence of phantom energy 

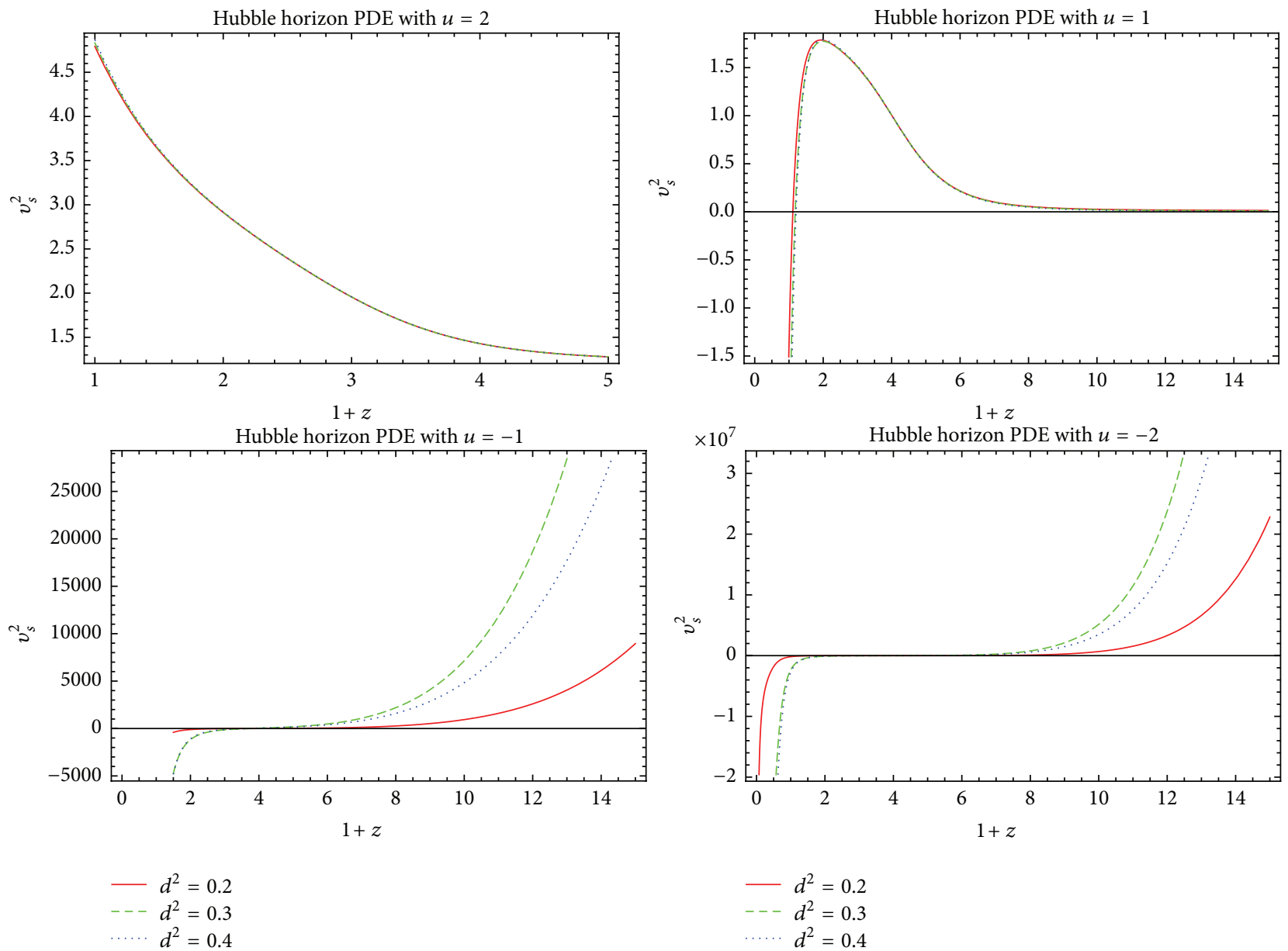

FIGURE 7: Plots of $v_{s}^{2}$ versus $1+z$ for Hubble horizon PDE model in dynamical Chern-Simons gravity with $u=2,1,-1,-2$.

for the interacting case is comparatively larger than the interacting case via EoS parameter. This model remained stable in different ranges depending on values of parameters while $\omega_{9}-\omega_{9}^{\prime}$ plane represented the same result as in [15]. In the present paper, we have obtained through the behavior of EoS parameter that the formation of BHs can be avoided through this framework of PDE with $u=2,-1,-2$. It is interesting to mention here that the ranges of EoS parameter for both PDE models lie within the observational constraints. For the $\omega_{9}-\omega_{9}^{\prime}$ plane for Hubble as well as event horizons $\mathrm{PDE}$, the $\Lambda \mathrm{CDM}$ limit is achieved for different values of PDE parameter $u$. The squared speed of sound represents stability at later epochs for both models.

\section{Concluding Remarks}

Up to now, most of the works on this gravity have been restricted to cosmological solutions of scale factor in flat FRW universe in the presence of family of well-known HDE models [31-33]. Moreover, the cosmological consequences have only been discussed in [34] for Ricci DE model without inclusion of DM. The purpose of this paper is to explore the cosmological implications in a well-known dynamical Chern-Simons modified gravity. The underlying DE model is
PDE with Hubble and event horizons along with interacting scenario with CDM. We have extracted the Hubble, EoS parameters, cosmological plane $\left(\omega_{9}-\omega_{9}^{\prime}\right)$, and squared speed of sound (for stability purpose). We have analyzed this cosmological setup through redshift, PDE, and interacting parameters.

Firstly, we have extracted the Hubble parameter for both PDE models. We have observed for Hubble horizon PDE that the evolution of Hubble parameter lies within the range $75 \pm 0.5$ for all values of interacting parameter for $u=2$ (upper left panel of Figure 1). However, Figure 1 shows that Hubble parameter lies in the range $(74,74.30)$ for all $d^{2}$ as well as for $u=1,-1,-2$ as shown in the right upper and lower panels, respectively. In case of event horizon PDE (Figure 3), the Hubble parameter remains in the range $74.80_{-0.005}^{+0.005}$ for $u=2$ (upper left panel). Moreover, the Hubble parameter increases with the passage of time for the cases $u=1,-1$. However, it lies between 73.4 and 74 for the case $u=-2$ (lower right panel). The precision of the cosmic distance scale has been greatly improved through two recent analyses. Our results of Hubble parameter show consistency with all observational constraints.

For Hubble horizon PDE, we have observed that the EoS parameter (Figure 2) exhibits the phantomlike behavior for 

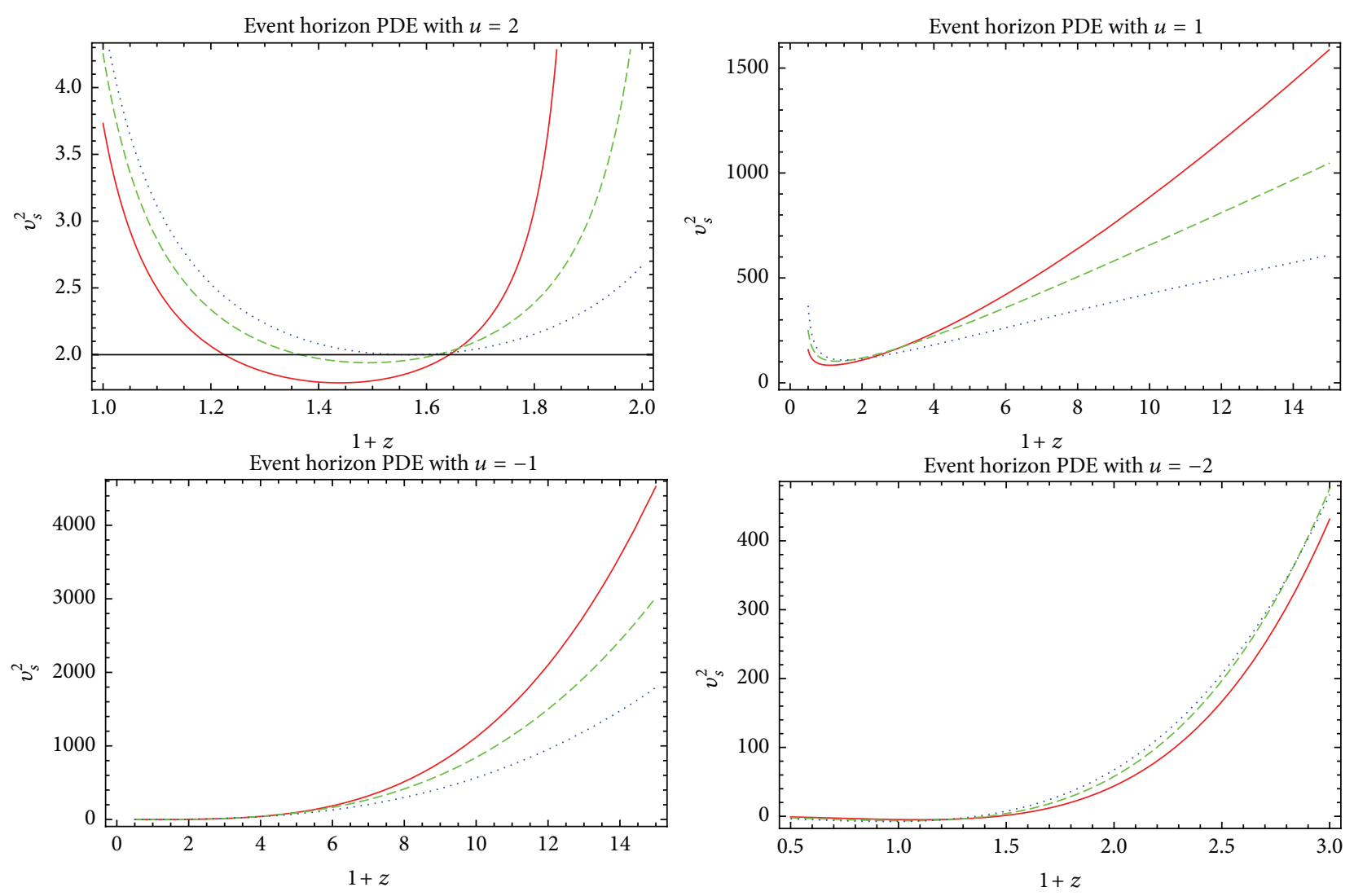

$$
\begin{aligned}
-d^{2} & =0.2 \\
---d^{2} & =0.3 \\
\cdots \cdots & d^{2}=0.4
\end{aligned}
$$$$
\begin{aligned}
- & d^{2}=0.2 \\
---d^{2} & =0.3 \\
\cdots & d^{2}=0.4
\end{aligned}
$$

FIgURE 8: Plot of $v_{s}^{2}$ versus $1+z$ for event horizon PDE in dynamical Chern-Simons modified gravity with $u=2,1,-1,-2$.

all cases of $u$ and $d^{2}$, except for the case of $u=1$ (where EoS shows variation from vacuum towards quintessence regions). Moreover, the EoS parameter lies in the ranges $-1.24 \leq \omega_{\vartheta} \leq$ -1 (for $u=2),-1 \leq \omega_{\vartheta} \leq-0.2$ (for $\left.u=1\right),-1.30 \leq \omega_{\vartheta} \leq-1$ (for $u=-1$ ), and $-2.30 \leq \omega_{9} \leq-1$ (for $u=-2$ ). In case of event horizon PDE, the present values of EoS parameter are $\omega_{90}=-0.98($ for $u=2), \omega_{90}=-1($ for $u=1), \omega_{90}=-0.88$ (for $u=-1$ ), and $\omega_{90}=-0.98$ (for $u=-0.78$ ). We also observed that all the trajectories of EoS parameter cross the phantom divide line from quintessence region and go towards phantom region in all the cases of $u=2,-1,-2$ except for $u=1$ case (where it starts from $\Lambda$ CDM limit and goes towards phantom era). However, the ranges of EoS parameter lie within the ranges of observational constraints. The fate of formation of $\mathrm{BHs}$ in the presence of phantomlike universe with large repulsive force has been discussed through EoS parameter, in detail. On the basis of the above discussion about the behavior of EoS parameter, we can conclude that the formation of $\mathrm{BHs}$ can be avoided through this framework of PDE with $u=2,-1,-2$. It is interesting to mention here that the ranges of EoS parameter for both PDE models lie within the following observational constraints.

We have also constructed the $\omega_{\vartheta}-\omega_{9}^{\prime}$ plane for Hubble as well as event horizons PDE with graphical presentation as given in Figures 5 and 6, respectively. For Hubble horizon PDE (Figure 5), the $\omega_{\vartheta}-\omega_{\vartheta}^{\prime}$ plane meets the $\Lambda$ CDM model, that is, $\left(\omega_{9}, \omega_{\vartheta}^{\prime}\right)=(-1,0)$, in all cases of $d^{2}$ for $u=1$ (right upper panel) and $u=-1$ (left lower panel). Also, the trajectories meet the thawing region (for $u=2,-1,-2$ ) and freezing region (for $u=1$ ). In case of event horizon PDE (Figure 6), the $\omega_{\vartheta}-\omega_{\vartheta}^{\prime}$ plane meets the $\Lambda$ CDM model for $u=-1$ only. Moreover, the trajectories meet the freezing region for all cases of $u$ and $d^{2}$. The constructed planes in both PDE models (Figures 5 and 6) also meet the observational constraints developed by Ade et al. [65].

The graphical behavior of squared speed of sound is displayed against redshift parameter in Figure 7 with the same constant parameters. We have observed from the plot (for $u=2$ ) that the squared speed of sound remains positive for the present as well as later epochs which exhibit the stability of the model. For the other case $u$, squared speed of sound exhibits the instability at the present epoch while it shows stability of the models for the later epoch. The plots of squared speed of sound are shown in Figure 8. Notice that it exhibits the viability as well as stability of the interacting PDE with event horizon in the present scenario throughout its history. 


\section{Conflict of Interests}

The authors declare that there is no conflict of interests regarding the publication of this paper.

\section{References}

[1] A. G. Riess, A. V. Filippenko, P. Challis et al., "Observational evidence from supernovae for an accelerating universe and a cosmological constant," The Astronomical Journal, vol. 116, no. 3, pp. 1009-1038, 1998.

[2] S. Perlmutter, G. Aldering, G. Goldhaber et al., "Measurements of $\Omega$ and $\Lambda$ from 42 high-redshift supernovae," The Astrophysical Journal, vol. 517, no. 2, p. 565, 1999.

[3] P. M. Garnavich, R. P. Kirshner, P. Challis et al., "Constraints on cosmological models from Hubble space telescope observations of high-z supernovae," The Astrophysical Journal, vol. 493, no. 2, pp. L53-L57, 1998.

[4] D. N. Spergel, L. Verde, H. V. Peiris et al., "First-year Wilkinson Microwave Anisotropy Probe (WMAP) observations: determination of cosmological parameters," The Astrophysical Journal Supplement Series, vol. 148, no. 1, p. 175, 2003.

[5] A. G. Riess, “Type Ia supernova discoveries at $z>1$ from the Hubble Space Telescope: evidence for past deceleration and constraints on dark energy evolution," The Astrophysical Journal, vol. 607, pp. 665-687, 2004.

[6] P. J. Peebles and B. Ratra, “The cosmological constant and dark energy," Reviews of Modern Physics, vol. 75, no. 2, pp. 559-606, 2003.

[7] G. F. Hinshaw, D. Larson, E. Komatsu et al., "Nine-year Wilkinson Microwave Anisotropy Probe (WMAP) observations: cosmological parameter results," The Astrophysical Journal Supplement Series, vol. 208, no. 2, p. 19, 2013.

[8] A. Y. Kamenshchik, U. Moschella, and V. Pasquier, "An alternative to quintessence," Physics Letters B, vol. 511, pp. 265-268, 2001.

[9] M. C. Bento, O. Bertolami, and A. A. Sen, "Generalized chaplygin gas, accelerated expansion, and dark-energy-matter unification," Physical Review D, vol. 66, Article ID 043507, 2002.

[10] X. Zhang, F. Q. Wu, and J. Zhang, "New generalized Chaplygin gas as a scheme for unification of dark energy and dark matter," Journal of Cosmology and Astroparticle Physics, vol. 1, article 3, 2006.

[11] S. D. H. Hsu, "Entropy bounds and dark energy," Physics Letters $B$, vol. 594, no. 1-2, pp. 13-16, 2004.

[12] M. Li, "A model of holographic dark energy," Physics Letters B, vol. 603, no. 1-2, pp. 1-5, 2004.

[13] R.-G. Cai, "A dark energy model characterized by the age of the universe," Physics Letters. B, vol. 657, no. 4-5, pp. 228-231, 2007.

[14] H. Wei, "Pilgrim dark energy," Classical and Quantum Gravity, vol. 29, no. 17, Article ID 175008, 2012.

[15] M. Sharif and A. Jawad, "Analysis of pilgrim dark energy models," The European Physical Journal C, vol. 73, no. 4, article 2382, 2013.

[16] M. Sharif and A. Jawad, "Pilgrim dark energy with apparent and event horizons in non-flat universe," The European Physical Journal C, vol. 73, no. 10, article 2600, 2013.

[17] A. Jawad, "Analysis of generalized ghost pilgrim dark energy in non-flat FRW universe," The European Physical Journal C, vol. 74, article 3215, 2014.
[18] M. Sharif and A. Jawad, "Analysis of generalized ghost version of pilgrim dark energy," Astrophysics and Space Science, vol. 351, no. 1, pp. 321-328, 2014.

[19] A. Jawad and A. Majeed, "Correspondence of pilgrim dark energy with scalar field models," Astrophysics and Space Science, vol. 356, no. 2, pp. 375-381, 2015.

[20] A. Jawad and S. Rani, "Cosmological evolution of pilgrim dark energy in $f(G)$ gravity," Advances in High Energy Physics, vol. 2015, Article ID 952156, 10 pages, 2015.

[21] A. Jawad and S. Rani, "Reconstruction of generalized ghost pilgrim dark energy in $F(\widetilde{R})$ gravity," Astrophysics and Space Science, vol. 359, article 23, 2015.

[22] L. Susskind, "The world as a hologram," Journal of Mathematical Physics, vol. 36, no. 11, article 6377, 1995.

[23] Y. J. Ng, "From computation to black holes and space-time foam," Physical Review Letters, vol. 86, no. 14, pp. 2946-2949, 2001.

[24] M. Arzano, T. W. Kephart, and Y. J. Ng, "From spacetime foam to holographic foam cosmology," Physics Letters. B, vol. 649, no. 4, pp. 243-246, 2007.

[25] X. Zhang and F. Q. Wu, "Constraints on holographic dark energy from type Ia supernova observations," Physical Review D, vol. 72, no. 4, Article ID 043524, 10 pages, 2005.

[26] A. G. Cohen, D. B. Kaplan, and A. E. Nelson, "Effective field theory, black holes, and the cosmological constant," Physical Review Letters, vol. 82, no. 25, pp. 4971-4974, 1999.

[27] R. Jackiw and S.-Y. Pi, "Chern-Simons modification of general relativity," Physical Review.D, vol. 68, Article ID 104012, 2003.

[28] J. Polchinski, Superstring Theory and Beyond String Theory, vol. 2 of Cambridge, UK, Cambridge University Press, 1998.

[29] A. Ashtekar, A. P. Balachandran, and S. Jo, "The CP problem in quantum gravity," International Journal of Modern Physics A, vol. 4, no. 6, p. 1493, 1989.

[30] S. Alexander and N. Yunes, "Chern-Simons modified general relativity," Physics Reports, vol. 480, no. 1-2, pp. 1-55, 2009.

[31] C. Furtado, J. R. Nascimento, A. Y. Petrov, and A. F. Santos, "Dynamical Chern-Simons modified gravity and FriedmannRobertson-Walkermetric," http://arxiv.org/abs/1005.1911.

[32] J. G. Silva and A. F. Santos, "Ricci dark energy in Chern-Simons modified gravity," The European Physical Journal C, vol. 73, article 2500, 2013.

[33] Y. S. Myung, "Comment on Ricci dark energy in Chern-Simons modified gravity," The European Physical Journal C, vol. 73, article 2515, 2013.

[34] A. Pasqua, R. da Rocha, and S. Chattopadhyay, "Holographic dark energy models and higher order generalizations in dynamical Chern-Simons modified gravity," The European Physical Journal C, vol. 75, article 44, 2015.

[35] D. Grumiller and N. Yunes, "How do black holes spin in ChernSimons modified gravity?" Physical Review D, vol. 77, Article ID 044015, 2008.

[36] P. Canizares, J. R. Gair, and C. F. Sopuerta, “Testing ChernSimons modified gravity with gravitational-wave detections of extreme-mass-ratio binaries," Physical Review D, vol. 86, Article ID 044010, 2012.

[37] K. Yagi, L. C. Stein, N. Yunes, and T. Tanaka, "Isolated and binary neutron stars in dynamical Chern-Simons gravity," Physical Review D, vol. 87, Article ID 084058, 2013.

[38] M. Sharif and A. Jawad, "Thermodynamics in closed universe with entropy corrections," International Journal of Modern Physics D, vol. 22, Article ID 1350014, 2013. 
[39] P. Martín-Moruno, "On the formalism of dark energy accretion onto black- and worm-holes," Physics Letters B, vol. 659, no. 1-2, pp. 40-44, 2008.

[40] M. Jamil, A. Qadir, and M. A. Rashid, "Charged black holes in phantom cosmology," The European Physical Journal C, vol. 58, no. 2, pp. 325-329, 2008.

[41] E. Babichev, S. Chernov, V. Dokuchaev, and Y. Eroshenko, "Ultrahard fluid and scalar field in the Kerr-Newman metric," Physical Review D, vol. 78, Article ID 104027, 2008.

[42] M. Jamil, "Evolution of a Schwarzschild black hole in phantomlike Chaplygin gas cosmologies," The European Physical Journal C, vol. 62, no. 3, pp. 609-614, 2009.

[43] M. Jamil and A. Qadir, "Primordial black holes in phantom cosmology," General Relativity and Gravitation, vol. 43, no. 4, pp. 1069-1082, 2011.

[44] J. Bhadra and U. Debnath, "Accretion of new variable modified Chaplygin gas and generalized cosmic Chaplygin gas onto Schwarzschild and Kerr-Newman black holes," The European Physical Journal C, vol. 72, article 1912, 2012.

[45] D. Pavón and W. Zimdahl, "Holographic dark energy and cosmic coincidence," Physics Letters B, vol. 628, no. 3-4, pp. 206210, 2005.

[46] W. Zimdahl and D. Pavón, "Interacting holographic dark energy," Classical and Quantum Gravity, vol. 24, article 5461, 2007.

[47] I. Durán, D. Pavón, and W. Zimdahlb, "Observational constraints on a holographic, interacting dark energy model," Journal of Cosmology and Astroparticle Physics, vol. 2010, no. 7, article 018, 2010.

[48] Y. Gong and T. Li, "A modified holographic dark energy model with infrared infinite extra dimension(s)," Physics Letters B, vol. 683, no. 4-5, pp. 241-247, 2010.

[49] A. Sheykhi, "Holographic scalar field models of dark energy," Physical Review D, vol. 84, Article ID 107302, 2011.

[50] Q. G. Huang and M. Li, "The holographic dark energy in a nonflat universe," Journal of Cosmology and Astroparticle Physics, vol. 2004, no. 8, article 013, 2004.

[51] M. Jamil, E. N. Saridakis, and M. R. Setare, "Holographic dark energy with varying gravitational constant," Physics Letters B, vol. 679, no. 3, pp. 172-176, 2009.

[52] M. Sharif and A. Jawad, "Modified holographic dark energy in non-flat Kaluza-Klein universe with varying G," The European Physical Journal C, vol. 72, article 1901, 2012.

[53] M. Sharif and A. Jawad, "Interacting modified holographic dark energy in Kaluza-Klein universe," Astrophysics and Space Science, vol. 337, no. 2, pp. 789-794, 2012.

[54] K. Karami, S. Ghaffari, and M. M. Soltanzadeh, "The generalized second law of gravitational thermodynamics on the apparent and event horizons in FRW cosmology," Classical and Quantum Gravity, vol. 27, Article ID 205021, 2010.

[55] M. R. Setare, "Interacting holographic dark energy model and generalized second law of thermodynamics in a non-flat universe," Journal of Cosmology and Astroparticle Physics, vol. 2007, no. 1, article 023, 2007.

[56] A. Sheykhi, "Thermodynamics of interacting holographic dark energy with the apparent horizon as an IR cutoff," Classical and Quantum Gravity, vol. 27, no. 2, Article ID 025007, 2010.

[57] N. Mazumder and S. Chakraborty, "Validity of the generalized second law of thermodynamics of the universe bounded by the event horizon in holographic dark energy model," General Relativity and Gravitation, vol. 42, no. 4, pp. 813-820, 2010.
[58] S. B. Wang, E. Abdalla, and R. K. Su, "Constraints on the dark energy from the holographic connection to the small $1 \mathrm{CMB}$ suppression," Physics Letters B, vol. 609, no. 3-4, pp. 200-205, 2005.

[59] X. Zhang and F. Q. Wu, "Constraints on holographic dark energy from type Ia supernova observations," Physical Review $D$, vol. 72, Article ID 043524, 2005.

[60] C. Feng, B. Wang, Y. Gong, and R.-K. Su, "Testing the viability of the interacting holographic dark energy model by using combined observational constraints," Journal of Cosmology and Astroparticle Physics, vol. 2007, no. 9, article 005, 2007.

[61] J. Lu, E. N. Saridakis, M. R. Setare, and L. Xu, "Observational constraints on holographic dark energy with varying gravitational constant," Journal of Cosmology and Astroparticle Physics, vol. 2010, no. 3, article 031, 2010.

[62] R. R. Caldwell and E. V. Linder, "Limits of quintessence," Physical Review Letters, vol. 95, Article ID 141301, 2005.

[63] A. G. Riess, L. Macri, S. Casertano et al., "A 3\% solution: determination of the Hubble constant with the Hubble space telescope and wide field camera 3," The Astrophysical Journal, vol. 730, no. 2, p. 119, 2011.

[64] W. L. Freedman, B. F. Madore, V. Scowcroft et al., "Carnegie Hubble program: a mid-infrared calibration of the Hubble constant," The Astrophysical Journal, vol. 758, no. 1, pp. 24-34, 2012.

[65] P. A. R. Ade, N. Aghanim, C. Armitage-Caplan et al., "Planck 2013 results. XVI. Cosmological parameters," Astronomy \& Astrophysics, vol. 571, article A16, 66 pages, 2014. 

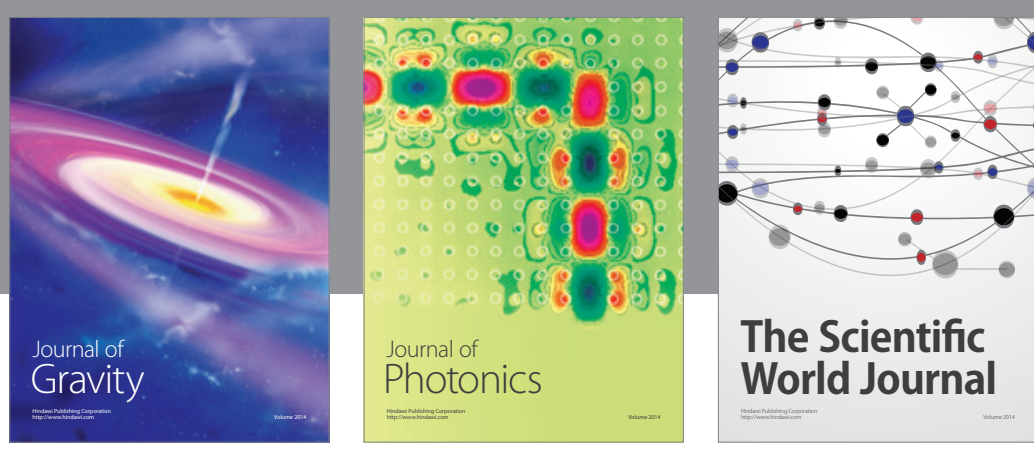

The Scientific World Journal
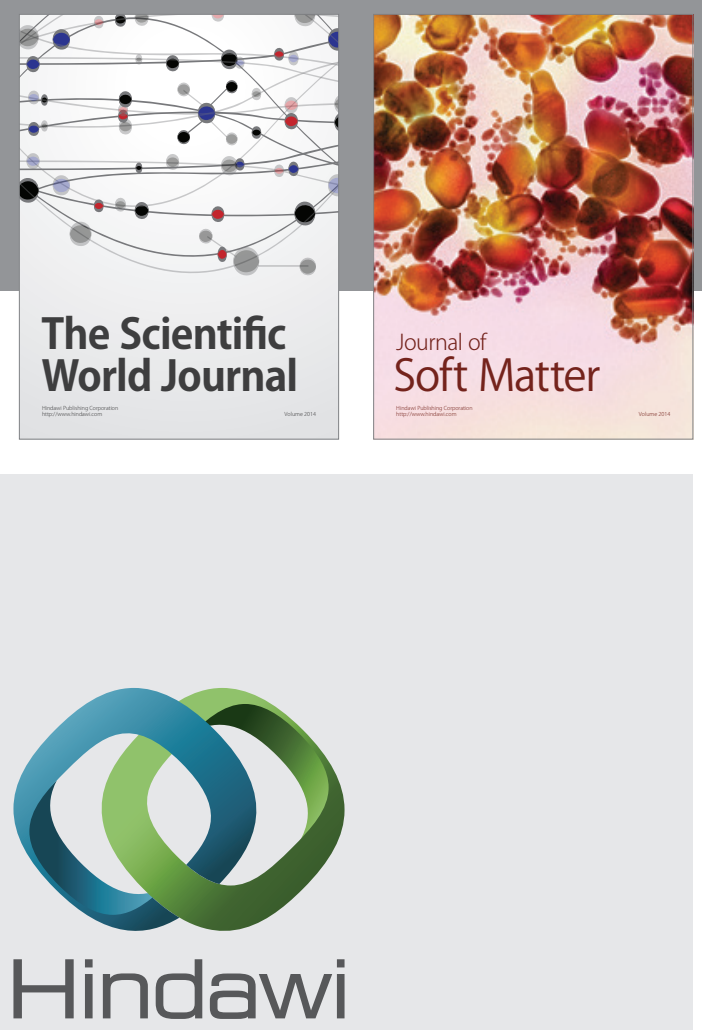

Submit your manuscripts at

http://www.hindawi.com

nternational Journal of

Statistical Mechanics
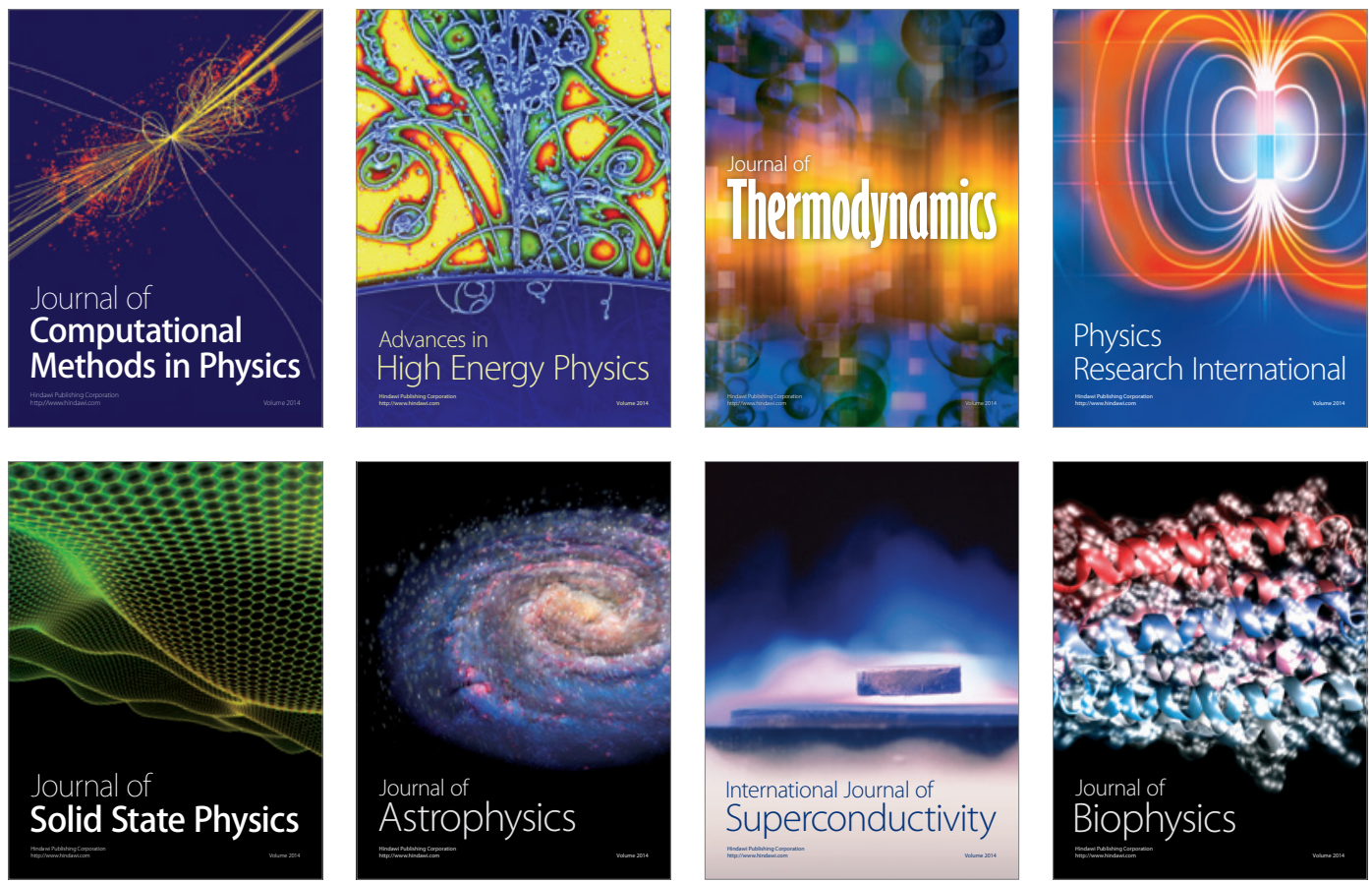
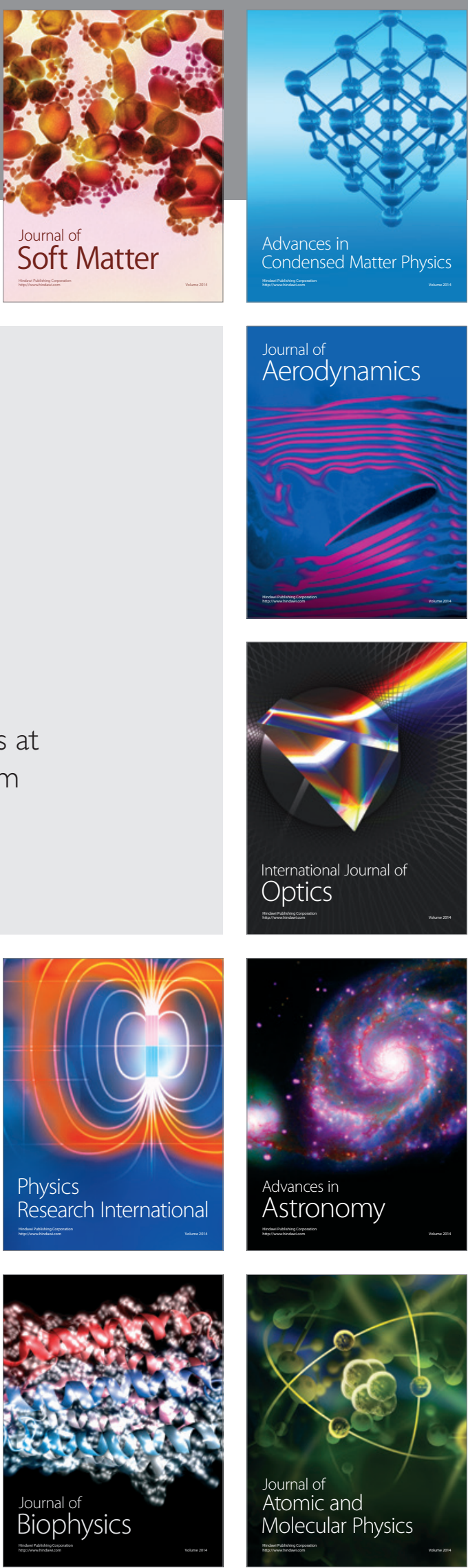